Courrier du Centre international Blaise Pascal

12 | 1990

Varia

\title{
Joubert Lecteur des Pensées de Pascal
}

\section{Thérèse Goyet}

\section{OpenEdition}

\section{Journals}

Édition électronique

URL : http://journals.openedition.org/ccibp/638

DOI : $10.4000 /$ ccibp. 638

ISSN : 2493-7460

\section{Éditeur}

Centre international Blaise Pascal

\section{Édition imprimée}

Date de publication : 4 janvier 1990

Pagination : 20-23

ISSN : 0249-6674

\section{Référence électronique}

Thérèse Goyet, « Joubert Lecteur des Pensées de Pascal », Courrier du Centre international Blaise Pascal [En ligne], 12 | 1990, mis en ligne le 08 janvier 2016, consulté le 22 avril 2019. URL : http:// journals.openedition.org/ccibp/638 ; DOI : 10.4000/ccibp.638

Ce document a été généré automatiquement le 22 avril 2019.

Centre international Blaise Pascal 


\title{
Joubert Lecteur des Pensées de Pascal
}

\author{
Thérèse Goyet
}

Reproduction de l'exemplaire des Pensées annoté par Joubert

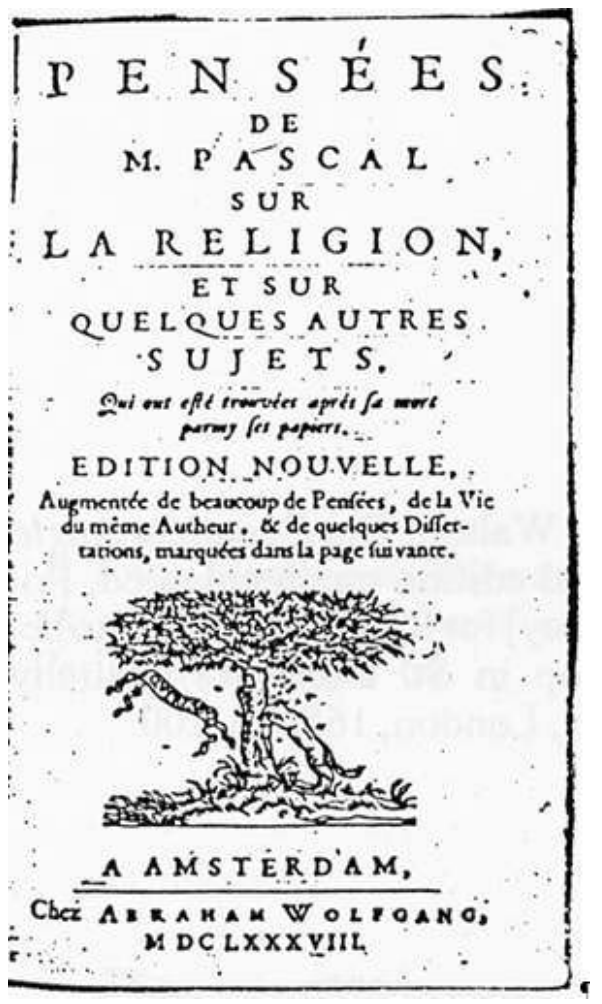

$\mathrm{BCIU}$

La Bibliothèque Municipale et Interuniversitaire de Clermont-Ferrand vient de faire, avec l'aide du Ministère de l'Éducation Nationale, l'acquisition d'un exemplaire des Pensées 
annoté par Joubert ${ }^{1}$. Il jouxtait dans la vente un exemplaire des Pensées de Leibnitz sur la Religion et la Morale, seconde édition, 2 vol. in- $8^{\circ}$, qui, lui, ne portait que la signature de son possesseur. Cet autre livre, voisin par le contenu thématique, était fort éloigné par le lieu et par la date: Paris, An XI, 1803. C'était donc une production contemporaine, au lieu que le Pascal faisait figure de livre ancien : À Amsterdam, chez Abraham Wolfgang, 1688.

2 Ce petit volume in-12 comporte : 48 pages, «La vie de M. Pascal » par sa sœur ; 56 pages non chiffrées de pièces diverses (Préface - celle de Port-Royal -, approbations, table des titres) : 242 pages qui sont proprement les «Pensées de M. Pascal sur la religion et sur quelques autres sujets » en 32 chapitres; plus une « Table des Matières » de 14 pages non chiffrées, qui est une sorte d'index thématique ; enfin une adjonction de 119 pages, qui apporte trois discours non signés mais que l'on sait être de Filleau de La Chaise. Cette édition de Hollande, diffusée en France sans privilège, n'apportait pas d'inédit: le texte des Pensées est celui de l'édition de Port-Royal de 1678, la Vie avait paru en 1684 à Amsterdam chez le même éditeur, et les discours avaient été publiés dès 1677 . La Bibliographie d'Albert Maire au t. IV, lui donne le $\mathrm{n}^{\circ} 34$, et nous désignerons l'exemplaire ayant appartenu à Joseph Joubert par la cote qui lui a été attribuée : R.5628.

\section{Pascal chez Joubert}

3 Avant que le rapport de Victor Cousin (1834) ait amené la critique à recourir au manuscrit autographe (édition Faugère, 1842), il n'y avait aucun inconvénient, quant au contenu, à lire les Pensées dans une édition du XVII ${ }^{\mathrm{e}}$ siècle. Joubert faisait ainsi l'économie des commentaires "philosophiques ». Le Pascal apologiste chrétien lui avait-il été mis en mains par ses éducateurs, les Pères de la Doctrine chrétienne, au collège de l'Esquile à Toulouse, où il fut entre 1768 et 1776 successivement élève, novice et professeur ? En fait nous ne savons rigoureusement rien de ses époques de lecture pascalienne, sauf un trait - qui n'a pas de rapport avec l'objet que nous considérons. (Il nous arrêtera tout à l'heure.) Quant aux traces que cette lecture aurait laissées dans son œuvre, il faudrait d'abord pouvoir parler d'une « œuvre » de Joubert. Cet «éducateur ${ }^{2}$ » des écrivains n'a jamais construit de livres. À ses amis il adressait des conseils et des remarques qu'on pourrait souvent qualifier de divinatoires; ses pensées, il les écrivait pour lui, quoique avec l'espoir qu'on les recueillerait. Sa condition d'écrivain présente donc quelque analogie avec celle de Pascal et justement ce sont des «Pensées » qu'après sa mort on tirera du journal intime contenu dans ses carnets. Comme on ne dispose pas encore d'une publication exhaustive ${ }^{3}$, les allusions à Pascal ne peuvent être comptées absolument, ni comparées en pourcentage aux occurrences des lectures d'autres écrivains. On peut cependant avancer que la présence de Pascal n'est pas très importante dans l'univers de Joubert.

4 Ce qui se montre à nous, c'est l'admiration en matière de goût et de rhétorique ${ }^{4}$ (la tradition d'ailleurs n'en a jamais été interrompue); quelques notations très communes concernent la biographie, les écrits et l'apologétique mise en œuvre ${ }^{5}$. Nous pouvons arrêter notre vue sur ce crayon bien affirmé :

Pascal. Altitude de cet esprit. On la voit derrière sa pensée. Sa fermeté. Exemption

de toute passion. C'est là surtout ce qui le rend très imposant ${ }^{6}$

5 Avouons que ce philosophe si noblement drapé nous étonne. Nous suggérerions que des lectures concomitantes ont à cet endroit projeté leurs reflets glorificateurs sur une représentation qui ne concorde pas avec les autres approches dont nous avons parlé. En 
effet Joubert a été un conseiller de Chateaubriand pour le Génie du Christianisme ${ }^{7}$. Mais en somme, faute de points de repère nombreux, le discret Joubert se dérobe à nos courbes et statistiques. Trouverons-nous au moins une rencontre typique dans les marges de notre R.5268?

\section{L'exemplaire annoté}

Sur le feuillet de garde au verso on trouve au crayon une petite table de remarques, qui renvoie aux pages du livre :

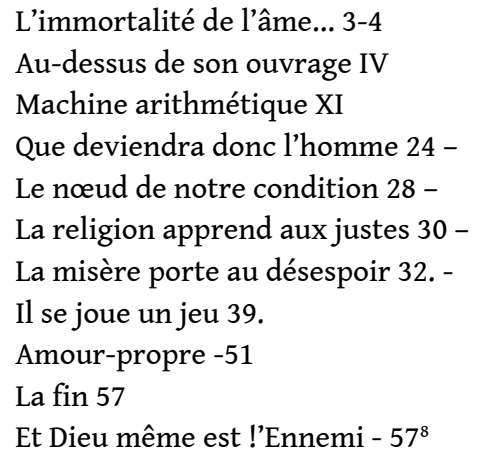

7 La liste ne mentionne pas tous les lieux du volume où se voit une intervention au crayon. C'était une habitude de Joubert d'annoter ainsi ses livres et l'on a même parlé de « hiéroglyphes ${ }^{9}$ ». Les signes ici ne sont pas très compliqués : lignes soulignées, traits ondulés à la marge ; croix ou signes d'appel variés dont le raccordement avec le texte est assez malaisé.

8 Ces marques, au cours de la Vie, sont relatives à l'éducation de Pascal, sa santé, ses mortifications (ex. p. 22, une ceinture de fer pleine de pointes). Il n'y en a pas dans la Préface (qui est celle d'Étienne Périer), et dans les Pensées elles s'arrêtent à la page 76, sur un signe à la marge, très appuyé, qui désigne, dans le chapitre XIV, Jésus-Christ, au fragment numéroté 5 , ce passage :

Mais quand ils [les Évangélistes] le font troublé, c'est quand il se trouble lui-même ;

et quand les hommes le troublent, il est tout fort.

Dans les premiers temps de l'église.

11 Pascal, p. 39, Chap. VII : "Qu'il est plus avantageux de croire que de ne pas croire ce qu'enseigne la Religion chrétienne. Au fr. 2. (C'est le texte que nous appelons « le pari», La. 418-Br. 233) :

Mais de quel côté pencherons-nous ? La raison dites-vous n'y peut rien déterminer. Il se joue un jeu à cette distance infinie, où il arrivera croix ou pile. Il y a un cahos ${ }^{11}$ infini qui nous sépare. Il se joue un jeu à cette distance infinie, où il arrivera croix ou pile. Que gagerezvous? Par raison vous ne pouvez assurer ny l'un ni l'autre? Par raison vous ne pouvez nier aucun des deux. ${ }^{12}$

12 Joubert, expliquant ce qu'il a souligné, complète la ligne :

La simple raison humaine.

Pascal, p. 42, même chapitre, même fragment : 
Suivez la manière par où ils ont commencé : imitez leurs actions extérieures. glemensw.)
S2. Penfées de M. Pascal.

$X$. avec l'un qu'il ne foit en guerre avec l'autre Ainf il eft toùjours divife \& contraire à luy même.

9. * Si c'elt un aveuglement qui n'eft pes naturel de vivre fans chercher ce qu'on et, c'en eft un encore bien plus terrible de vivar mal en croyant Dieu. Tousles hommes pref que font dans l'un ou dans l'autre de ces deur aveuglemens. + Stuat íncis, incusti!

$\mathrm{BCIU}$

Pascal, p. 58, Chap. X, « Juifs », au fr. 13 :

Dieu avait tellement disposé les choses que tout servait à le faire reconnaître. [Jésus-Christ, dans son premier avènement]. Les Juifs le prouvoient en le recevant; car ils estoient les dépositaires des prophéties, et ils le prouvoient aussi en ne le recevant point, parce qu'en cela ils accomplissoient les prophéties.

Joubert avec un trait vertical à la marge, note :

Argument cornu.

La signification de ces deux mots interjetés dépend du registre de langue que Joubert s'est choisi. S'il entend parler comme tout le monde, c'est-à-dire préférant l'expressivité à l'exactitude, le jugement est défavorable, voire méprisant. Si l'on en croit Littré, une absurdité au sujet de cornes, mise en syllogisme, a fixé la dépréciation. L'emploi par dérision remonte au moins au XVII ${ }^{\mathrm{e}}$ siècle $^{14}$. Seulement c'est une dégradation. Pour trouver les origines du syntagme il faut remonter à la terminologie scolastique. Littré le dit au cours de l'article cornu :

On appelait aussi de ce nom le dilemme, parce qu'il frappe des deux côtés ( argumentum cornutum feriens utrinque $)^{15}$. 
Or Joubert a reçu une excellente formation philosophique et il a continué toute sa vie à lire les philosophes, discutant de Kant lu dans le latin, et lui-même disciple éclairé de Platon ${ }^{16}$. Il ne fait donc pas de doute qu'en ce point de sa lecture de Pascal il a voulu marquer à son usage qu'il avait reconnu le procédé du dilemme.

De soi le dilemme est un raisonnement dur. Il peut être vicieux, dit la Logique de Port-Royal, "quand la disjonction sur laquelle il est fondé, est défectueuse, ne comprenant pas tous les membres du tout que l'on divise ${ }^{17}$ ». Et justement les éditeurs port-royalistes de Pascal avaient bien eu conscience que ce dilemme-ci serait difficile à comprendre (et qu'il ne fallait pas ajouter à la répugnance qu'on aurait à l'admettre). Dans la construction qu'ils ont instaurée Joubert rencontre un Pascal intégré, en ce chapitre "Juifs", à un développement qui roule sur la continuité de l'attente du Messie, la fameuse "perpétuité ». Mais la construction d'après l'ordre de la Copie F.fr. 9 203, dont Lafuma a été le principal instigateur, déplace la perspective. On est dans un chapitre XIX, «Loi figurative» dont le thème principal est l'impossibilité pour le cœur enténébré d'appréhender la réalité spirituelle. De plus les éditeurs qui ont recouru au texte original, ayant d'ailleurs reconnu deux fragments là où Port-Royal n'en faisait qu'un, nous donnent à lire aujourd'hui :

Que pouvaient faire les Juifs, ses ennemis ? S'ils le reçoivent-ils le prouvent par leur réception, car les dépositaires de l'attente du Messie le recevaient, et s'ils le renoncent, ils le prouvent par leur renonciation ${ }^{18}$.

La mise au temps présent accentue par sa généralité le caractère logique ; «renoncer » est aussi plus violent que «ne pas recevoir », et le mot « renonciation » n'était pas clair. Les éditeurs de Port-Royal l'avaient donc remplacé par une explication et Joubert lisait une phrase ajoutée :

... parce qu'en cela ils accomplissoient les prophéties.

Sous ces modérations apportées par des éditeurs timides il avait cependant décelé l'armature implacable.

\section{L'énigme des références}

27 Notre R. 5268 fait un joli livre mais sa reliure est modeste. Plaisait-il particulièrement à son possesseur? Est-il de ceux qu'il se plaisait à cirer de sa main gantée ${ }^{19}$ ? Il ne fut sûrement pas le seul exemplaire des Pensées à être entré dans sa bibliothèque. À la date du 8 septembre 1813, Joubert relève des expressions relatives au travail littéraire, et, chose tout à fait exceptionnelle chez ce lecteur insoucieux du travail critique, il donne des références aux pages. Mais celles-ci ne correspondent point aux signes d'attention portés dans notre exemplaire ni au contenu desdites pages dans l'édition de $1688^{20}$.

Et en un autre lieu il fait à ses propres occupations autour de Pascal une allusion qui nous embarrasse fort :

Voyez le manuscrit de ses Pensées que j'ai vu pour la première fois le samedi 26 , revu et lu le lundi 28 mars de cette année ${ }^{21}$.

Le manuscrit? Quel manuscrit? À supposer que Joubert ait été familier de la Bibliothèque Nationale - qui va bientôt devenir Bibliothèque Impériale - il aurait pu, par une curiosité alors inouïe - « voir » le Recueil original, notre F. fr. 9202 ; il aurait pu le « revoir » mais le « lire ", et en un jour? Et si c'était ce recueil qu'il a eu dans les mains, comment n'auraitil pas été frappé par sa singularité, et par la difficulté de son déchiffrage? 

appeler « le manuscrit des Pensées » ? Un de nos amis va nous en parler, sans pour autant faire la clarté. Il s'agit d'Antoine-Augustin Renouard (1765-1853), légataire de l'abbé Bossut auquel on doit la première édition des œuvres scientifiques de Pascal. C'est à Renouard que nous devons la transmission du manuscrit Usage de la Machine ${ }^{22}$. En 1803 précisément, il donnait lui-même une édition des Pensées ${ }^{23}$ où il reprend le texte, de l'édition Bossut, mais en l'améliorant, surtout dans le second volume. Or il réfère les corrections qu'il introduit tantôt « aux manuscrits », tantôt au « manuscrit original », et ce dernier renvoi touche bien exactement dans le recueil original ${ }^{24}$. Or il existe deux copies faites du temps de l'édition de Port-Royal ${ }^{25}$. Ce qui devrait faire, trois manuscrits. Renouard en mentionnant deux connaissait donc, avec l'autographe, une des Copies. Mais Renouard était un grand philologue, habitué aux investigations critiques.

31

Joubert était un grand lecteur, insoucieux des débats sur la lettre des textes. Faudra-t-il penser que, fréquentant les milieux de la conservation érudite, il se sera fait montrer le manuscrit des Pensées, c'est-à-dire celui qui était le plus lisible, c'est-à-dire la plus connue des Copies? Ou bien, s'il ne lisait que chez lui, il se sera chargé de revoir un manuscrit mis au point en vue d'une édition des Pensées en gestation? Rien n'atteste qu'il ait connu Renouard, mais rien ne l'empêche non plus.

Il est bon et salutaire qu'une collecte de témoignages se termine par une impossibilité de décider. Du moins avons-nous vérifié que Joubert ne fut pas un acteur capital dans la fortune des Pensées. Mais son regard attentif nous permet de voir sourdre, dans l'appel spiritualiste qui marque le début du XIX ${ }^{\mathrm{e}}$ siècle et qui fit le succès du Génie du Christianisme , le besoin de lire Pascal.

\section{NOTES}

1. Vente de la Bibliothèque Jacques Guérin, à Paris, Drouot-Montaigne, en date du 29 novembre $1988, n^{\circ} 9$ du catalogue.

2. Voir Rémy Tessonneau, Joubert éducateur (1754-1824) d'après des documents inédits, Paris, Plon, 1944.

3. Joubert est généralement lu dans des anthologies. Nous nous réfèrerons à l'édition la plus complète : Les Carnets de Joseph Joubert, Textes recueillis sur les manuscrits autographes par André Beaunier, [publication posthume], Paris, Gallimard, 1938, 2 vol. L'éditeur signale souvent qu'il a laissé tomber certains passages. La première édition (Recueil des Pensées de M Joubert, Paris, impr. de Le Normant, 1838 , in- $8^{\circ}$ ) est due à Chateaubriand lui-même. Il avait opéré sur une copie réalisée par un neveu de Joubert, Jean-Baptiste Michel Duchesne, et il avait pris des libertés auprès desquelles celles des éditeurs « pascalins » de Port-Royal font figure d'exactitude critique. Nous avons puisé bien des renseignements dans les Actes du colloque Joubert, Villeneuve-sur-Yonne, 31 mai, $1^{\text {er }}$ et 2 juin 1985, [diffusion: Paris, Nizet, 1986], particulièrement dans Rémy Tessonneau, "Connaissance de Joubert », pp. 9-21, et Jean Campagnac, « Joubert et les livres », pp. 97-105.

4. Carnets, 2 avril 1803, p. 376 ; 8 janvier 1805, p. 191 ; 20 janvier 1810, p. 755. 
5. « Malade », 20 mars 1802, Carnets, p. 340 ; «misanthropie chrétienne », 24 juillet 1805, p. 510 ; «n'a rien inventé », 24 juillet 1805, p. 510 ; « des pensées de Montaigne », 28 janvier 1808, p. 640 ; les gestes qui « inclinent la machine », la piété « un anéantissement de soi », 23 avril 1799, p. 203.

6. Carnets, p. 340, à la date du 22 avril 1800.

7. Voir Tessonneau, Joubert éducateur, p.108-109, et Maurice Regard, dans l'édition du Génie du Christianisme, La Pléiade, 1978, pp. 1594-1601.

8. Disposition, orthographe et ponctuation, strictement conservées. Cette table n'a point été transcrite dans le catalogue de vente. Il semble qu'elle ne soit pas de la main de Joubert. Les trois premières lignes renvoient à la Vie par $\mathrm{M}^{\mathrm{me}}$ Périer (qui d'ailleurs est paginée en chiffres arabes) ; les suivantes au contenu des Pensées.

9. J. Champagnac, dans Colloque, p. 101.

10. Pages $20,39,42,52,58$. Relevées dans le catalogue, sauf celle de la p. 52 .

11. Sic. Joubert corrige en chaos.

12. Les soulignements dans nos citations sont toujours les siens.

13. La difficulté dans l'ordre grammatical est de savoir si l'adjectif " incerti » est l'attribut de " tous les hommes ", nous renvoyant à la phrase de Pascal par un saut d'anacoluthe, ou s'il tombe sur « fines» voisin, de manière exclamative.

Ce latin de philosophe nous semble dépendre du vocabulaire de Cicéron, De finibus bonorun et malorun - titre si difficile à traduire ! - et des discussions scolastiques.

14. Littré. Cornu, 2. Argument cornu. Exemples tirés de Condillac et de Scarron. Reproduits à Dilemme par Le Grand Robert de la Langue française. Littré donne pourtant une définition neutre : «Terme de logique. Sorte d'argument. » Robert au mot Cornu infère : « Vieux. Argument faux » et «Par analogie : Raisons, idées, visions cornues = Extravagant, biscornu, bizarre. » L'adjectif cornu, qui évoque le diable entre dans beaucoup d'expressions dépréciatives.

15. Id. Foulquié, Dictionnaire de la langue philosophique, $3^{\mathrm{e}}$ éd. 1978, à Dilemme : « Les scolastiques l'appelaient syllogismus comutus ».

16. Voir Colloque cité, p. 105, 140, 141 ; les Carnets, passim et notamment p. 396, 28 août 1803. "Son plan [de Pascal] est dramatique. Il y a usé de la circulaire et du contour platonicien ». Cf. Patricia A. Ward, Joseph Joubert and the critical tradition : platonism and romanticism, Genève, Droz, 1980.

17. La logique ou l'Art de penser, $3^{\mathrm{e}}$ partie, Chap. 16. Les auteurs de Port-Royal ne parlent pas du tout du « cornu».

18. La. 262 - Br. 762.

19. Colloque cité, p. 104.

20. Carnets, t II, pp. 755-756, 8 septembre 1813. Pascal cherchait « les tours... capables de frapper l'esprit et d'intéresser le cœur » (page XV), « se renfermait dans l'essentiel et le simple naturel » ( ibid.), «composa ses lettres avec une contention d'esprit, un soin et travail extrême " (à commencer par la sixième). «Il était quelquefois vingt jours entiers sur une seule lettre; il en recommençait quelques-unes jusqu'à sept ou huit fois. On dit même qu'il reprit la dix-huitième jusqu'à treize fois " (page XXI). - Nous n'avons pas retrouvé le lieu d'où sont extraites ces citations.

21. Carnets, p. 376, 2 avril 1803.

22. Voir Courrier du CIBP, $\mathrm{n}^{\circ} 4,1982$, pp. 6-11, Thérèse Goyet, «Le manuscrit "Usage de la machine"», et Courrier, n 8, 1986, le texte publié par Guy Mourlevat et Dominique Descotes.

23. Pensées de Blaise Pascal, Paris, chez Antoine Augustin Renouard, XI = 1803, 2 vol. Nous n'avons rien trouvé qui précise la période de parution dans l'année.

24. Ed. Renouard, t. II, p. 230 à 235, donne quatre "glanures » qui améliorent le texte, soit $\mathrm{CX}=$ La. 554 - Br. 303 ; CX IV = 604-871; CXVIII = 809-230; CXIX = 161-221. C'est pour cette dernière pensée qu'il « se croit obligé de la réimprimer ici telle que Pascal l'a écrite, page 63 du manuscrit original ». Le renvoi est bien exact. 
25. Voir Jean Mesnard, « Aux origines de l'édition des Pensées: les deux Copies », dans Les Pensées de Pascal ont trois cents ans, Clermont-Ferrand, G. de Bussac, 1971, p. 1-30.

\section{AUTEUR}

\section{THÉRÈSE GOYET}

Directeur-adjoint du CIBP, membre fondateur 